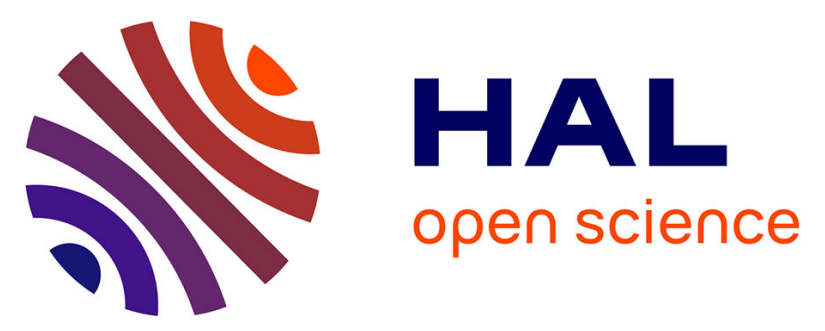

\title{
Tumor-size responses to first-line is a predictor of overall survival in metastatic colorectal cancer
}

\author{
Lola-Jade Palmieri, Amina Fihri, Solène Doat, Olivier Dubreuil, Gilles \\ Manceau, Mehdi Karoui, Mathilde Wagner, Olivier Lucidarme, Jean-Baptiste \\ Bachet
}

\section{To cite this version:}

Lola-Jade Palmieri, Amina Fihri, Solène Doat, Olivier Dubreuil, Gilles Manceau, et al.. Tumor-size responses to first-line is a predictor of overall survival in metastatic colorectal cancer. European Radiology, 2019, 29 (7), pp.3871-3880. 10.1007/s00330-018-5967-0 . hal-02173155

\section{HAL Id: hal-02173155 \\ https://hal.sorbonne-universite.fr/hal-02173155}

Submitted on 4 Jul 2019

HAL is a multi-disciplinary open access archive for the deposit and dissemination of scientific research documents, whether they are published or not. The documents may come from teaching and research institutions in France or abroad, or from public or private research centers.
L'archive ouverte pluridisciplinaire HAL, est destinée au dépôt et à la diffusion de documents scientifiques de niveau recherche, publiés ou non, émanant des établissements d'enseignement et de recherche français ou étrangers, des laboratoires publics ou privés. 
Tumor-size responses to first-line is a predictor of overall survival in metastatic colorectal cancer

Lola-Jade Palmieri $^{1,4, *}$ • Amina Fihri ${ }^{2,4, *}$. Solène Doat ${ }^{1,4}$ • Olivier Dubreuil ${ }^{1,4}$. Gilles Manceau $^{3,4} \cdot$ Mehdi Karoui ${ }^{3,4}$ - Mathilde Wagner ${ }^{2,4,5} \cdot$ Olivier Lucidarme $^{2,4,5}$. Jean-Baptiste Bachet ${ }^{1,4}$

${ }^{1}$ Gastroenterology and Digestive Oncology Department, Pitié-Salpêtrière Hospital, Assistance Publique-Hôpitaux de Paris, Paris, France

${ }^{2}$ Radiology Department, Pitié-Salpêtrière Hospital, Assistance Publique-Hôpitaux de Paris, Paris, France

${ }^{3}$ Digestive Surgery Department, Pitié-Salpêtrière Hospital, Assistance Publique-Hôpitaux de Paris, Paris, France

${ }^{4}$ Sorbonne University, Paris, France

${ }^{5}$ INSERM, CNRS, UMR7371-U1146, Laboratoire d'Imagerie Biomédicale, 75006 Paris, France

* These authors contributed equally to this work.

$\bowtie$ Olivier Lucidarme, Radiology Department, Pitie-Salpêtrière University Hospital, 47-83, boulevard de l'Hôpital, 75651 Paris Cedex 13, France. Tel: +33 (0)1 421767 94; Fax: +33 (0)1 421782 24; e-mail: olivier.lucidarme@aphp.fr 
Tumor-size responses to first-line is a predictor of overall survival in metastatic colorectal cancer

\begin{abstract}
(/250)
Objectives Early tumor shrinkage (ETS) has been reported to be associated with survival of metastatic colorectal cancer (mCRC) patients. Our aim was to analyze long-term tumor-size evolution, according to early $\mathrm{mCRC}$ best responses during the first-line therapy, to evaluate first best response--survival links.
\end{abstract}

Methods Sixty-five patients with unresectable mCRCs, treated between 2010 and 2015, were included retrospectively in this descriptive monocenter study, and grouped according to their RECIST 1.1 first-line best responses: progressive disease $\left(\mathrm{PD}_{\mathrm{fl}}\right)$, stable disease with tumorsize evolution between 0 and $+19 \%\left(\mathrm{SD}_{\mathrm{fl}}+\right)$ or 0 and $-29 \%\left(\mathrm{SD}_{\mathrm{fl}}-\right)$, and partial responders (PRs), who were classed PR with ETS $\left(\mathrm{ETS}_{\mathrm{fl}}\right)$ or without $\left(\mathrm{PR}_{\mathrm{fl}}\right)$. Tumor-size evolution and best tumor responses to each chemotherapy line were analyzed.

Results Tumor loads of $\mathrm{ETS}_{\mathrm{fl}}$ or $\mathrm{PR}_{\mathrm{fl}}$ mCRCs tended to remain inferior to their initial values: $60 \%$ of patients died with target-lesion sums below baseline. For first-line $\mathrm{SD}_{\mathrm{fl}}+$ or $\mathrm{PD}_{\mathrm{fl}}$ mCRCs, rapid tumor-load increases continued during successive lines: $>80 \%$ died with target-lesion sums above baseline. ETS $_{\mathrm{fl}}$ mCRCs responded better to subsequent lines $(37.5 \%$ second-line PR), whereas $\mathrm{PD}_{\mathrm{fl}}$ mCRCs remained refractory to other therapies ( $0 \%$ secondand third-line PR). Overall survival rates were significantly $(p=0.03)$ longer for the $\mathrm{ETS}_{\mathrm{fl}}$ group (29.9 [95\% CI: 12.6-47.1] months); shorter for the $\mathrm{PD}_{\mathrm{fl}}$ group (17.1 [95\% CI: $1.5-$ 37.5] months).

Conclusion Tumors responding to first-line chemotherapy also responded better to subsequent lines, whereas $\mathrm{PD}_{\mathrm{fl}}$ mCRCs remained refractory, which may explain the better survival associated with $\mathrm{ETS}_{\mathrm{fl}}$. 
Online electronic supplemental material is available for this article.

Keywords Response Evaluation Criteria in Solid Tumors, Colonic Neoplasms, Survival rate, Tumor Burden.

\section{Key points}

- Early shrinking tumors under first-line chemotherapy responded better to subsequent lines, maintaining low tumor loads, potentially explaining the link between early tumor shrinkage and overall survival of metastatic colorectal cancer ( $m C R C)$ patients.

- $\quad$ mCRCs progressing under first-line chemotherapy remained refractory to other therapies and their tumor loads increased rapidly.

- Even outside a clinical trial, an early first CT-scan reevaluation with RECIST criteria 8 weeks after starting first-line therapy is crucial to determine long-term mCRC evolution.

\section{Abbreviations:}

CT: computed tomography

EGFR: epidermal growth-factor receptor

ETS: early tumor shrinkage

ETS $_{\mathrm{fl}}$ : early tumor shrinkage at first line (defined at 8 weeks)

fl: first line

mCRC: metastatic colorectal cancer

OS: overall survival 
PFS: progression-free survival

PD: progressive disease

$\mathrm{PD}_{\mathrm{fl}}$ : progressive disease at first line

PR: partial response

$\mathrm{PR}_{\mathrm{fl}}$ : partial response at first line (without $\mathrm{ETS}_{\mathrm{fl}}$ : delayed response)

RECIST: Response Evaluation Criteria In Solid Tumors

SD: stable disease

$\mathrm{SD}_{\mathrm{fl}}:$ stable disease at first line 


\section{Introduction}

Metastatic colorectal cancer (mCRC) management now achieves longer overall survival (OS), reaching 30 months in recent phase III trials [1-4]. For fit patients, first-line treatments are based on doublet- or triplet-chemotherapy combined with a targeted agent. Anti-angiogenic bevacizumab is approved for all patients, whereas panitumumab and cetuximab, which target the epidermal growth-factor receptor (EGFR), are limited to the subgroup of patients with $R A S$ (a gene mutation predictive of the non-efficacy of anti-EGFR) wild-type mCRC [5]. At present, the choice of first-line regimen is guided by $R A S$ status and the aim of obtaining tumor shrinkage for patients with symptomatic disease or potentially resectable metastases [5].

In general, clinical trials evaluate chemotherapy efficacy by OS, progression free-survival (PFS) and tumor objective response rate (ORR). PFS is often used as a surrogate criterion for OS, as an early endpoint. Tumor response is evaluated by ORR, assessed with revised Response Evaluation Criteria in Solid Tumors (RECIST criteria (version 1.1) [6]) that clinicians also use extensively in routine practice to adapt their therapeutic strategies-

The validity of PFS as a surrogate endpoint for OS in $\mathrm{mCRC}$ is questioned [7, 8], as median OS now exceeds 30 months, but PFS is still about 10 months. In addition, in a phase III trial that compared doublet-chemotherapy plus anti-EGFR or bevacizumab (FIRE-3) [3], median PFS did not differ between arms, whereas an OS benefit in favor of anti-EGFR was still found. One of the hypotheses to explain this OS-PFS discordance is the efficacy of subsequent chemotherapy lines. Another hypothesis is that other response-related outcome parameters, such as early tumour shrinkage (ETS) and depth-of-response, could have made an OS difference, despite comparable PFS [9]. ETS and depth-of-response are indicators of precociousness and intensity of tumor-size response to therapy, and predictors of OS and PFS $[10,11]$. ETS (depending on its definition) is achieved when the tumor load has decreased 
$10-30 \%$ [12], as measured at the time of first imaging control, between 6 to 8 weeks after starting treatment. Depth-of-response is defined as the percentage of tumor shrinkage, based on the sum of the longest diameters, observed at the time of the lowest value (nadir) compared to before starting therapy (baseline). ETS and depth-of-response have since been validated retrospectively by several prospective trials' results [11, 13-17].

The tumor-size evolution in response to first-line therapy (henceforth initial response) has been particularly studied as a predictor of OS and PFS [15, 18], but its impact on responses to subsequent treatment lines has never been investigated. Only radiological data on first-line therapy were available in those retrospective analyses. Hence, evolution under successive lines remains hypothetical [12].

Our aim was to analyze tumor-size evolution over the long-term, according to the early mCRC initial responses, to explore the link between initial tumor-size evolution and OS.

\section{Methods}

\section{Patients}

Ethics Committee approval was waived for this single-center retrospective study.

All the patients treated in our center for unresectable mCRC, between 01/01/2010 and $30 / 01 / 2015$, were identified by computer search of the chemotherapy-prescription software. We included only conventional adenocarcinomas (excluding mucinous tumors) treated by cytotoxic and targeted chemotherapies. None of them were treated with immuno therapy. Patients with at least one measurable lesion, according to the RECIST 1.1 criteria $[6,19]$ were included. Target lesions were defined at baseline and the largest diameter had to be $\geq 10$ $\mathrm{mm}$ or short axis $\geq 15 \mathrm{~mm}$ if the lesion was a lymph node. 
We excluded patients: 1) with no available baseline computed-tomography (CT) scan and patients with fewer than five reevaluation CT scans; 2) with no target lesions according to RECIST 1.1 criteria or with target lesions that could not be followed over the long term, especially after secondary metastatic liver resection; and 3) without sufficient clinical followup information (on different chemotherapy lines or outcome).

\section{Image analysis and clinical information}

All, but 10, baseline scans and all follow-up scans were performed in our institution using two CT systems, with 2x128 detector-rows (ICT 256, Philips healthcare) or with 128 detectorrows (ICT 128, Philips healthcare). The acquisition were done according to the actual standard, without and after an intravenous injection of $2 \mathrm{ml} / \mathrm{kg}$ body weight of an iodinated contrast agent $(2.5 \mathrm{ml} / \mathrm{s})$ at $350 \mathrm{mg}$ of iodine/ml, during the arterial phase $(35 \mathrm{~s}$, slice thickness: $1.5 \mathrm{~mm})$ at the thoracic level, and during the portal phase (70 s; slice thickness: 2 $\mathrm{mm}$ ) at the abdominal and pelvic levels. Tumor-size evolution was evaluated by CT scan according to RECIST criteria [6], with a first-evaluation at 8 weeks, then every 8-12 weeks. RECIST criteria are based on the largest target-lesion diameters combined with a subjective assessment of non-target lesions. Target lesions are defined on the baseline CT scan. On follow-up images, the target-lesion response is evaluated as the percentage change of the sum of their largest diameters against baseline or subsequent scan with the lowest sum of target disease (nadir). The ORR is defined as the sum of complete (disappearance of all target lesions) and partial responses (PR, $\geq 30 \%$ decrease of the sum of target-lesion diameters, compared to baseline). Progressive disease (PD) is $\geq 20 \%$ increase of the sum of target-lesion diameters, with the smallest study sum (including baseline) serving as the reference. Stable disease (SD) is neither response nor PD.

The first CT scan analyzed was always the baseline examination just before first-line 
therapy. A maximum of five target lesions could be chosen, with a maximum of two per organ. When analyzing a follow-up scan, the same target lesions were measured and their sum was again calculated. Two radiologists (O.L. and A.F., with 25 and 2 years of experience, respectively), blinded to each patient's clinical findings and previous radiology reports, retrospectively analyzed the $\mathrm{CT}$ images by consensus. Response-to-therapy was assessed manually using the PACS Carestream (Carestream Health).

Clinical information (patient characteristics, tumor characteristics, chemotherapy lines, responses to chemotherapy and outcome) was collected from each patient's electronic or paper medical chart by the oncologist (L.-J.P.).

\section{Analytical groups}

Patients were separated into five groups according to their best responses to first-line (fl) chemotherapy, defined as follows: $\mathrm{ETS}_{\mathrm{fl}}, \geq 30 \%$ tumor reduction at 8 weeks; $\mathrm{PR}_{\mathrm{fl}}, \geq 30 \%$ tumor reduction but without $\mathrm{ETS}_{\mathrm{fl}}$ (delayed response); $\mathrm{SD}_{\mathrm{fl}}+$ or $\mathrm{SD}_{\mathrm{fl}}-$, respectively, with tumor-size evolution between +1 and $+19 \%$ or 0 and $-29 \%$; and $\mathrm{PD}_{\mathrm{fl}}: \geq 20 \%$ tumor growth and/or appearance of new lesion(s). Figure 1 is an example of radiological evaluation.

\section{Long-term tumor-size evolution}

For each patient, we used RECIST criteria customized for this study as follows: first, we focused only on the target lesions, not taking into account the non-target lesions. Second, the largest diameters of the initial target lesions on every reevaluation CT scan, from baseline to death or end of follow-up, were summed. Target lesions were not modified when chemotherapy changed. To display the results, we considered the sum of the first line 
baseline CT scan's target lesions to be $100 \%$ and the evolution of the sum of the largest diameter(s) at each of the subsequent CT scans, regardless of different chemotherapy lines, was systematically assessed as a percentage of variation from that first line baseline $100 \%$. To avoid, as much as possible, bias induced by the appearance of new lesions, we systematically added $20 \%$ to the curve when new lesions were identified. We choose arbitrarily $20 \%$ because this increase defines progression according to the Recist criteria as the appearance of new lesion does. Finally, to easily visualize deaths and to distinguish nonsurvivors from survivors or censored in one readable graph, we arbitrarily represented death visually as top line on the graph reached by each patient's curve. We analyzed the tumor burden (the sum of target lesions) at endpoint, defined as the last reevaluation CT scan.

\section{Response to each new chemotherapy line}

In a second analysis, the evolution of the sum of the largest diameters at the time of the best response was systematically assessed as a percentage of variation from the corresponding baseline for each new chemotherapy line. Each baseline was thus considered 100\%, independently from the baselines of the previous chemotherapy lines. Hence, graphically, a return to $100 \%$ means a treatment-line change. This analytical methodology yielded a "sawtoothed" line for each patient. Each new treatment line was defined as a change of chemotherapy or targeted agents. Unlike the analysis of the tumor-size evolution over the long term, death is not represented on the graph. Maintenance therapies and reintroduction of the first-line cytotoxic agent(s) after progression under maintenance were not considered a new line. We evaluated second- and third-line ORRs, defined as best responses to therapy (sum of complete and PRs) and the disease-control rate (sum of complete responses, PRs and SDs). 


\section{Statistical analyses}

Quantitative variables are summarized using descriptive statistics: number of patients with available data $(n)$ or mean \pm standard deviation; and compared with ANOVA test. Qualitative variables are expressed by arm as $n(\%)$ and compared with chi ${ }^{2}$ test with Yates correction for small sample sizes.

Survival rates, estimated with the Kaplan-Meier method, are expressed as median (95\% confidence interval (CI)) duration, and compared using the log-rank test. OS lasted from the date of the first administration of the first-line regimen to that of death (all causes). PFS was defined as the time from the date of the first administration of the first-line regimen and to that of progression or death. Survivors without progression were censored on the date of the most recent information up-date. The cut-off date for the final data analysis was November $15,2016$.

Statistical analyses were computed with $\mathrm{R}$ software, with statistical significance set at $p$ value $<0.05$

\section{Results}

\section{Population studied}

Among the 226 potentially eligible patients (Figure 2), 161, whose lesions could not be followed over the long term, were excluded; the final analysis concerned the 65 patients with complete radiological and clinical follow-up information available. According to the radiological review, patients' best responses at first line were scored as follows: 14/65 (22\%) ETS $_{\mathrm{fl}}, 18 / 65(28 \%) \mathrm{PR}_{\mathrm{fl}}, 19 / 65(29 \%) \mathrm{SD}_{\mathrm{fl}}-$, 8/65 (12\%) $\mathrm{SD}_{\mathrm{fl}}+$ and 6/65 (9\%) $\mathrm{PD}_{\mathrm{fl}}$. 
The global characteristics of the 65 patients and according to their five groups, defined by their initial tumor ORRs, are described in Table 1. No significant differences were found between the five groups for sex ratio, age, primary tumor and the metastatic sites, mutational status, tumor differentiation, and the type of first-line therapy. Only the microsatellite instability (MSI) status distribution differed significantly among the five groups $(p=0.02)$, with the ETS $_{\mathrm{fl}}$ group having more microsatellite stability (MSS).

\section{Long-term tumor-size evolution and clinical outcomes}

Spider plots of individual patient's tumor-size evolution, appearance of new lesions over the long-term, and death as assessed with our customized RECIST criteria, according to initial best response group, are represented in Figure 3. Clinical outcomes (Death, OS, PFS) and tumor burdens at endpoint and death are reported in Table 2.

Among the patients with $\mathrm{PD}_{\mathrm{fl}}$ or $\mathrm{SD}_{\mathrm{fl}}+, 12 / 14(86 \%)$ had target-lesion sums superior to their first baseline assessment at endpoint (dead or alive), as opposed to 18/51 (35\%) patients with $\mathrm{ETS}_{\mathrm{fl}}$ or $\mathrm{PR}_{\mathrm{fl}}$ or $\mathrm{SD}_{\mathrm{fl}}-(p<0.001)$. Considering death as the only endpoint, 11/12 (92\%) patients with $\mathrm{PD}_{\mathrm{fl}}$ or $\mathrm{SD}_{\mathrm{fl}}+$ died with lesion sums superior to their initial baseline, whereas for patients with $\mathrm{ETS}_{\mathrm{fl}}, \mathrm{PR}_{\mathrm{fl}}$ or $\mathrm{SD}_{\mathrm{fl}}-$, only 15/37 (41\%) did $(p<0.01)$.

OS also differed significantly among groups $(p=0.03)$, being longer for the $\operatorname{ETS}_{\mathrm{fl}}$ group (median OS: 29.9 (95\% CI: 12.6-47.1) months) and shorter for the $\mathrm{PD}_{\mathrm{fl}}$ group (median OS: 17.1 (95\% CI: 1.5-37.5) months). Median PFS lasted 15 (95\% CI: 9.8-24.2) months for the ETS $_{\mathrm{fl}}$ group vs 2.9 (95\% CI: 4.9-15.3) months for the $\mathrm{PD}_{\mathrm{fl}}$ group, however the betweengroup difference did not reach significance $(p=0.3)$.

\section{Response to each new chemotherapy line}


Individual patient's best responses to each chemotherapy line, according to his/her initial response, are represented in Figure 4. Response and disease-control rates to second- and third-line therapies are detailed for each initial response group in Table 3. Visually, tumor responses to successive lines differed substantially, depending on the initial response. Patients with $\mathrm{ETS}_{\mathrm{fl}}$ had more negative peaks below baseline $100 \%$ and $\mathrm{SD}_{\mathrm{fl}}+$ or $\mathrm{PD}_{\mathrm{fl}}$ patients had more positive peaks above that baseline for successive treatments. In other words, patients with an initial response to first-line chemotherapy tended to have a response to the following lines of chemotherapy, whereas patients who progressed initially tended to also not respond to following lines. This tendency was not statistically significant due to the small number of patients in each group.

\section{Discussion}

Assessing tumor responses with ETS and depth-of-response was recently shown to be associated with better OS and PFS outcomes [12], but only responses to first-line therapy were reported. Herein, we focused on the impact of the initial responses on long-term outcomes. Two mCRC-evolution types were distinguished. First, $\mathrm{mCRCs}$ with $\mathrm{ETS}_{\mathrm{fl}}, \mathrm{PR}_{\mathrm{fl}}$ or $\mathrm{SD}_{\mathrm{fl}}-$, which maintained low tumor loads (only $41 \%$ patients died with tumor sizes superior to baseline), had better responses to subsequent therapy lines. Their initial tumor ORR was significantly associated with longer OS. Indeed, that beneficial response to first-line chemotherapy seemed to be prolonged over time, under successive lines. Second, mCRCs with $\mathrm{PD}_{\mathrm{fl}}$ or $\mathrm{SD}_{\mathrm{fl}}+$, whose tumor loads rose $(91 \%$ died with a target-lesion sums superior to their baseline assessment), continued to be mostly refractory to later therapy lines.

Our results are in accordance with those of the analysis of the role of tumor-size reduction in response to first-line therapy as a predictor of mCRC patients' long-term outcomes [12, 14]. ETS, defined $\mathrm{a} \geq 20 \%$ or $30 \%$ response, was proved to be associated with better PFS and 
OS after doublet-chemotherapy with an anti-EGFR [9, 13, 15, 17, 20, 21], and after bevacizumab with triplet- or double-chemotherapy [14]. In the literature, patients with ETS, compared to those without, had maximum $\triangle \mathrm{PFS}$ of 7.6 months and maximum $\Delta \mathrm{OS}$ of 33.8 months. Those findings are summarized in Table E1 (online). We also described tumor-size evolution after this early response to chemotherapy, which had not been studied previously. Even outside a clinical trial, an early first CT-scan reevaluation with RECIST criteria between 6 and 8 weeks seems crucial to determine long-term mCRC evolution. Notably, we found that the initial response might be suggestive of future responses to later lines of therapy and, hence, longer OS and PFS.

Explanations that can be put forward are: on the one hand, initial intrinsic tumor aggressiveness remains constant or worsens, regardless of the chemotherapy prescribed. Indeed, changing the chemotherapy did not visibly impact the factors responsible for that aggressiveness. On the other hand, less aggressive tumors maintain a favorable profile in response to different types of chemotherapy, even after resistant-clone selection by the previous lines. This observation suggests that some aggressiveness factors are independent, or unaffected, by the different chemotherapy lines currently being used. This hypothesis raises the question of whether or not to pursue chemotherapy for mCRCs with initial progression at the first CT reevaluation at 8 weeks. Indeed, these tumors with immediate progression, which represent $10 \%$ of $\mathrm{mCRCs}$, do not seem respond to the successive chemotherapies and have very little chance of later disease control. This issue should be evaluated prospectively, comparing best supportive care alone to best supportive care plus chemotherapy, but conducting such a study is complicated, for ethical reasons. Our study has some limitations. First, our single-center study included a relatively small number of patients, only 65, but all had fully documented clinical and radiological (at least five reevaluation scans) follow-up. Indeed, we excluded many potential subjects because CT 
scans were not available to analyze the evolution from first scan to death. Our study population was, however, quite representative of mCRCs with, at the first reevaluation: 49.2\% $\mathrm{ETS}_{\mathrm{fl}}$ or $\mathrm{PR}_{\mathrm{fl}}$ responders, $41.5 \% \mathrm{SD}_{\mathrm{fl}}$ and $9.2 \% \mathrm{PD}_{\mathrm{fl}}$, rates similar to those published previously $[2,3]$. Also, the five groups' characteristics were quite similar. Second, the representation of new lesions is another potential limitation. Indeed, we chose to represent new lesions by systematically adding $20 \%$ to each patient's curve, which could be too much or too little. In addition, we did not consider non-target lesions, even though we know that the tumor burden sometimes increases due to the non-target lesions and not because of preexisting target lesions that can become necrotic or fibrotic. Hence, following the same lesion through the different chemotherapy lines may underestimate the true tumor-burden evolution. However, that bias would be similar for all groups, and does not explain the differences we found. Third, we did not analyze tumor-size responses according to treatment regimen for the different groups, because heterogeneous chemotherapies were used and more patients would be required to obtain meaningful information. Fourth, we decided arbitrarily to split into two parts the RECIST "stable disease" group. Our aim was to consider separately the outcome of all the patients with an initial positive slope for their lesion size evolution (ie, $\mathrm{PD}_{\mathrm{fl}}$ and $\mathrm{SD}_{\mathrm{fl}}+$ ) and those with a negative slope (ie, $\mathrm{SD}_{\mathrm{fl}}, \mathrm{PR}_{\mathrm{fl}}$ and $\left.\mathrm{ETS}_{\mathrm{fl}}\right)$. However even if our results suggest that the SD group gather patients with different evolutive profiles, this is based on a small cohort and further studies are needed to confirm this point.

Fifth tumor response assessment was done by two radiologists by consensus, consequently no interobserver agreement can be known, however we did not study or use non-validated methods to assess the tumor responses.

Analyses of long-term tumor-size evolutions according to early response to first-line chemotherapy could be applied to larger populations in multiple centers with mathematical modeling of those evolutions. In addition, in the near future, we should be able to assess 
tumor evolution reliably and even earlier with circulating tumor DNA. Indeed, an early change of the latter's concentration could be marker of therapeutic efficacy in mCRC patients, as recently assessed in two prospective studies $[22,23]$.

In conclusion, a response to first-line chemotherapy was associated with the selection of chemosensitive mCRCs, future responses to subsequent treatment lines and longer OS. An initial lack of response or $\mathrm{PD}_{\mathrm{fl}}$ was associated with tumors refractory to successive therapy lines with rising tumor loads. Tumor-size evolution according to initial response to first line chemotherapy may thus predict survival. These findings reinforce the interest of an early tumor reevaluation, 8 weeks after baseline $\mathrm{CT}$ assessment, to predict long-term mCRC evolution.

\section{References}

1. Cremolini C, Loupakis F, Antoniotti C et al (2015) FOLFOXIRI plus bevacizumab versus FOLFIRI plus bevacizumab as first-line treatment of patients with metastatic colorectal cancer: updated overall survival and molecular subgroup analyses of the openlabel, phase 3 TRIBE study. Lancet Oncol 16:1306-1315.

2. Schwartzberg LS, Rivera F, Karthaus M et al (2014) PEAK: a randomized, multicenter phase II study of panitumumab plus modified fluorouracil, leucovorin, and oxaliplatin (mFOLFOX6) or bevacizumab plus mFOLFOX6 in patients with previously untreated, unresectable, wild-type KRAS exon 2 metastatic colorectal cancer. J Clin Oncol 32:22407.

3. Heinemann V, von Weikersthal LF, Decker T et al (2014) FOLFIRI plus cetuximab versus FOLFIRI plus bevacizumab as first-line treatment for patients with metastatic colorectal cancer (FIRE-3): a randomised, open-label, phase 3 trial. Lancet Oncol 15:1065-1075.

4. Venook AP, Niedzwiecki D, Lenz H-J et al (2017) Effect of first-line chemotherapy combined with cetuximab or bevacizumab on overall survival in patients with KRAS wild-type advanced or metastatic colorectal cancer: a randomized clinical trial. JAMA 317:2392-2401.

5. Van Cutsem E, Cervantes A, Adam R et al. (2016) ESMO consensus guidelines for the 
management of patients with metastatic colorectal cancer. Ann Oncol 27:1386-422.

6. Eisenhauer EA, Therasse P, Bogaerts J et al (2009) New response evaluation criteria in solid tumours: revised RECIST guideline (version 1.1). Eur J Cancer 45:228-247.

7. Venook AP, Tabernero J. (2015) Progression-free survival: helpful biomarker or clinically meaningless end point? J Clin Oncol 33:4-6.

8. Claret L, Gupta M, Han K, et al. (2013) Evaluation of tumor-size response metrics to predict overall survival in western and Chinese patients with first-line metastatic colorectal cancer. J Clin Oncol 31:2110-2114.

9. Stintzing S, Modest DP, Rossius L et al (2016) FOLFIRI plus cetuximab versus FOLFIRI plus bevacizumab for metastatic colorectal cancer (FIRE-3): a post-hoc analysis of tumour dynamics in the final $R A S$ wild-type subgroup of this randomised open-label phase 3 trial. Lancet Oncol 17:1426-1434.

10. Piessevaux H, Buyse M, De Roock W et al. (2009) Radiological tumor size decrease at week 6 is a potent predictor of outcome in chemorefractory metastatic colorectal cancer treated with cetuximab (BOND trial). Ann Oncol 20:1375-1382.

11. Suzuki C, Blomqvist L, Sundin A et al (2012) The initial change in tumor size predicts response and survival in patients with metastatic colorectal cancer treated with combination chemotherapy. Ann Oncol 23:948-954.

12. Heinemann V, Stintzing S, Modest DP et al (2015) Early tumour shrinkage (ETS) and depth of response $(\mathrm{DpR})$ in the treatment of patients with metastatic colorectal cancer (mCRC). Eur J Cancer 51:1927-1936.

13. Piessevaux H, Buyse M, Schlichting M et al (2013) Use of early tumor shrinkage to predict long-term outcome in metastatic colorectal cancer treated with cetuximab. J Clin Oncol 31(30):3764-3775.

14. Cremolini C, Loupakis F, Antoniotti C et al. (2015) Early tumor shrinkage and depth of response predict long-term outcome in metastatic colorectal cancer patients treated with first-line chemotherapy plus bevacizumab: results from phase III TRIBE trial by the Gruppo Oncologico del Nord Ovest. Ann Oncol 26:1188-1194.

15. Douillard J-Y, Siena S, Peeters M et al (2015) Impact of early tumour shrinkage and resection on outcomes in patients with wild-type $R A S$ metastatic colorectal cancer. Eur $\mathrm{J}$ Cancer 51:1231-1242.

16. Giessen C, Laubender RP, Fischer von Weikersthal L et al (2013) Early tumor shrinkage in metastatic colorectal cancer: retrospective analysis from an irinotecan-based randomized first-line trial. Cancer Sci 104:718-724. 
17. Modest DP, Laubender RP, Stintzing S et al (2013) Early tumor shrinkage in patients with metastatic colorectal cancer receiving first-line treatment with cetuximab combined with either CAPIRI or CAPOX: an analysis of the German AIO KRK 0104 trial. Acta Oncol 52:956-962.

18. Petrelli F, Pietrantonio F, Cremolini C et al (2015) Early tumour shrinkage as a prognostic factor and surrogate end-point in colorectal cancer: a systematic review and pooled-analysis. Eur J Cancer 51:800-807.

19. Therasse P, Arbuck SG, Eisenhauer EA et al (2000) New guidelines to evaluate the response to treatment in solid tumors. J Natl Cancer Inst 92:205-216.

20. Tsuji A, Sunakawa Y, Ichikawa W et al (2016) Early tumor shrinkage and depth of response as predictors of favorable treatment outcomes in patients with metastatic colorectal cancer treated with FOLFOX plus cetuximab (JACCRO CC-05). Target Oncol 11:799-806.

21. Rivera F, Karthaus M, Hecht JR et al (2017) Final analysis of the randomised PEAK trial: overall survival and tumour responses during first-line treatment with mFOLFOX6 plus either panitumumab or bevacizumab in patients with metastatic colorectal carcinoma. Int J Colorectal Dis 32:1179-1190.

22. Tie J, Kinde I, Wang Y, et al (2015) Circulating tumor DNA as an early marker of therapeutic response in patients with metastatic colorectal cancer. Ann Oncol 26:17151722 .

23. Garlan F, Laurent-Puig P, Sefrioui D et al (2017) Early evaluation of circulating tumor DNA as marker of therapeutic efficacy in metastatic colorectal cancer patients (PLACOL study). Clin Cancer Res doi: 10.1158/1078-0432.CCR-16-3155 


\section{Legends}

Table 1. Patient characteristics and treatments received according to first-line chemotherapyresponse group.

Table 2. Overall survival (OS), progression-free survival (FPS) and tumor burdens at endpoint and death.

Table 3. Response and disease-control rates after second- and third-line treatments, according to initial tumor-response group.

Figure 1. A 68-year-old patient with pT4N1M+ rectal adenocarcinoma and synchronous bilobar liver metastases and peritoneal carcinomatosis a) Baseline CT scan before first-line therapy (Folfox + Bevacizumab): example of a target lesion across the upper part of the $2^{\text {nd }}$, $4^{\text {th }}$ and $8^{\text {th }}$ segments of the liver . b) CT scan before the second-line therapy (LV5FU2 + Bevacizumab): Progressive disease with tumor increasing of 30\%. C) CT scan before the third-line therapy (Folfiri + Bevacizumab): Progressive disease due to appearance of new lesions in the peritoneum (arrow). d) CT scan before the fourth-line therapy (Folfiri + Aflibercept): Progressive disease with tumor increasing of $31 \%$ compared to c).

Figure 2. Flow chart. $L 1 C 1$, line 1 cycle 1, ETS early tumor shrinkage, $P R$ partial response, $S D$ stable disease tumor-size evolution 8 weeks after starting first-line $\left({ }_{\mathrm{fl}}\right)$ chemotherapy between 0 and $+19 \%\left(\mathrm{SD}_{\mathrm{fl}}+\right)$ or 0 and $-29 \%\left(\mathrm{SD}_{\mathrm{fl}}-\right)$, or $P D$ progressive disease.

Figure 3. Individual patient's global tumor-size evolution over the long-term, as assessed with RECIST 1.1 criteria, including new lesions, represented by systematically adding $20 \%$ to the curve, according to the initial first-line $\left({ }_{\mathrm{fl}}\right)$ chemotherapy-response group: ETS early tumor shrinkage, $P R$ partial response, $S D$ stable disease tumor-size evolution between 0 and $+19 \%$ $\left(\mathrm{SD}_{\mathrm{fl}}+\right)$ or 0 and $-29 \%\left(\mathrm{SD}_{\mathrm{fl}}-\right)$, or $P D$ progressive disease, expressed as the percentage variation from the baseline $100 \%$ with return to that visual level. 
Figure 4. Responses expressed as the percentage of variation from the baseline of each new chemotherapy line. Each baseline was thus considered 100\%, independently from the baselines of the previous chemotherapy lines. On the graph, a return to $100 \%$ means a treatment-line change. Each graph provides the responses to each new chemotherapy line for each initial first-line (fl) best response group: $E T S_{f l}$ early tumor shrinkage, $P R_{f l}$ partial response, $S D_{f l}$ stable disease tumor-size evolution between 0 and $+19 \%\left(S D_{f l}+\right)$ or 0 and $29 \%\left(S D_{f \vdash}\right)$, or $P D_{f l}$ progressive disease. 
Table 1 Patient characteristics and treatments received according to first-line chemotherapy-response group

\begin{tabular}{|c|c|c|c|c|c|c|}
\hline Characteristic & Total & $\mathrm{ETS}_{\mathrm{fl}}$ & $\mathrm{PR}_{\mathrm{fl}}$ & $\mathrm{SD}_{\mathrm{fl}-}$ & $\mathrm{SD}_{\mathrm{fl}}+$ & $\mathrm{PD}_{\mathrm{fl}}$ \\
\hline Number of patients & $65(100 \%)$ & $14(21.5 \%)$ & $18(27.7 \%)$ & $19(29.2 \%)$ & $8(12.3 \%)$ & $6(9.2 \%)$ \\
\hline \multicolumn{7}{|l|}{ Sex } \\
\hline Women & $22(33.8 \%)$ & $5(35.7 \%)$ & $8(44.4 \%)$ & $6(31.6 \%)$ & $3(37.5 \%)$ & - \\
\hline Men & $43(66.2 \%)$ & $9(64.3 \%)$ & $10(55.6 \%)$ & $13(68.4 \%)$ & $5(62.5 \%)$ & $6(100 \%)$ \\
\hline \multicolumn{7}{|l|}{ Age (years) } \\
\hline$<65$ & $29(44.6 \%)$ & $5(35.7 \%)$ & $6(33.3 \%)$ & $10(52.6 \%)$ & $3(37.5 \%)$ & $5(83.3 \%)$ \\
\hline$\geq 65$ and $\leq 70$ & $15(23.1 \%)$ & $4(28.6 \%)$ & $5(27.8 \%)$ & $5(26.3 \%)$ & - & $1(16.7 \%)$ \\
\hline$>70$ & $21(32.3 \%)$ & $5(35.7 \%)$ & $7(38.9 \%)$ & $4(21.1 \%)$ & $5(62.5 \%)$ & - \\
\hline \multicolumn{7}{|l|}{ Laboratory values } \\
\hline Serum albumin $(\mathrm{g} / \mathrm{L})$ & $34.86 \pm 5.3$ & $35.5 \pm 5.3$ & $35.6 \pm 4.1$ & $33.8 \pm 5.3$ & $35.5 \pm 9.2$ & $33.4 \% \pm 4.7$ \\
\hline Lactate dehydrogenase (IU/L) & $571.8 \pm 399.4$ & $460.8 \pm 191.7$ & $703.7 \pm 420.1$ & $684.4 \pm 540.7$ & $326.2 \pm 142.2$ & $465.5 \pm 178.9$ \\
\hline Serum alkaline phosphate (IU/L) & $217.4 \pm 313.9$ & $210.9 \pm 400.1$ & $207.4 \pm 231.8$ & $294.4 \pm 411.5$ & $154.1 \pm 178.3$ & $98.8 \pm 8.1$ \\
\hline \multicolumn{7}{|l|}{ Primary tumor site } \\
\hline Right colon & $18(27.7 \%)$ & $2(14.3 \%)$ & $6(33.3 \%)$ & $7(36.8 \%)$ & $3(37.5 \%)$ & - \\
\hline Left colon & $31(47.7 \%)$ & $8(57.1 \%)$ & $12(66.7 \%)$ & $7(36.8 \%)$ & - & $4(66.7 \%)$ \\
\hline Rectum & $16(24.6 \%)$ & $4(28.6 \%)$ & - & $5(26.3 \%)$ & $5(62.5 \%)$ & $2(33.3 \%)$ \\
\hline \multicolumn{7}{|l|}{ Mutational status } \\
\hline WT $R A S / B R A F$ & $18(27.7 \%)$ & $5(35.7 \%)$ & $6(33.3 \%)$ & $2(10.5 \%)$ & $3(37.5 \%)$ & $2(33.3 \%)$ \\
\hline Mutated $R A S$ & $31(47.7 \%)$ & $5(35.7 \%)$ & $8(44.4 \%)$ & $11(57.9 \%)$ & $3(37.5 \%)$ & $4(66.7 \%)$ \\
\hline Mutated $B R A F$ & $2(3.1 \%)$ & - & $1(5.5 \%)$ & - & $1(12.5 \%)$ & - \\
\hline Unknown & $14(21.5 \%)$ & $4(28.6 \%)$ & $3(16.7 \%)$ & $6(31.6 \%)$ & $1(12.5 \%)$ & - \\
\hline \multicolumn{7}{|l|}{ MSI status } \\
\hline MSS & $39(60 \%)$ & $12(85.7 \%)$ & $10(55.6 \%)$ & $7(36.8 \%)$ & $4(50 \%)$ & $6(100 \%)$ \\
\hline MSI & $7(10.8 \%)$ & - & $1(5.6 \%)$ & $2(10.5 \%)$ & $4(50 \%)$ & - \\
\hline Unknown & $19(29.2 \%)$ & $2(14.3 \%)$ & $3(16.7 \%)$ & $10(52.6 \%)$ & - & - \\
\hline \multicolumn{7}{|l|}{ Tumor differentiation } \\
\hline Poorly differentiated & $4(6.2 \%)$ & $2(14.3 \%)$ & - & $1(5.2 \%)$ & - & $1(16.7 \%)$ \\
\hline Moderately differentiated & $34(52.3 \%)$ & $10(71.4 \%)$ & $10(55.6 \%)$ & $7(36.8 \%)$ & $5(62.5 \%)$ & $2(33.3 \%)$ \\
\hline Well-differentiated & $16(24.6 \%)$ & $2(14.3 \%)$ & $5(27.8 \%)$ & $6(31.6 \%)$ & $2(25 \%)$ & $1(16.7 \%)$ \\
\hline Unknown & $11(16.9 \%)$ & - & $3(16.7 \%)$ & $5(26.3 \%)$ & $1(12.5 \%)$ & $2(33.3 \%)$ \\
\hline \multicolumn{7}{|l|}{ Type of metastases } \\
\hline Synchronous & $40(61.5 \%)$ & $10(71.4 \%)$ & $14(77.8 \%)$ & $12(63.2 \%)$ & $1(12.5 \%)$ & $3(50 \%)$ \\
\hline Metachronous & $25(38.5 \%)$ & $4(28.6 \%)$ & $4(22.2 \%)$ & $7(36.8 \%)$ & $7(87.5 \%)$ & - \\
\hline
\end{tabular}


Resection of primary tumor

Yes (before metastatic chemotherap 42 (64.6\%)

Yes (during metastatic chemotherap 11 (16.9\%)

No

$12(18.5 \%)$

$12(85.7 \%)$

$8(44.4 \%)$

$7(87.5 \%)$

$5(83.3 \%)$

$1(7.1 \%)$

$5(27.8 \%)$

$1(12.5 \%)$

$1(16.7 \%)$

Metastatic sites

Liver not affected

$(7.1 \%)$

$5(27.8 \%)$

$6(31.6 \%)$

$-$

Liver only

$9(13.8 \%)$

Liver and others

Number of metastatic sites

1

$24(36.9 \%)$

$4(28.6 \%)$

$-$

$3(15.8 \%)$

$2(25 \%)$

$8(44.4 \%)$

$7(36.8 \%)$

$1(12.5 \%) \quad 5(83.3 \%)$

$32(49.2 \%)$

$7(50 \%)$

$10(55.6 \%)$

$9(47.4 \%)$

$5(62.5 \%)$

$1(16.7 \%)$

$32(49.2 \%) \quad 7(50 \%)$

$23(35.4 \%) \quad 4(28.6 \%)$

$8(44.4 \%)$

$9(47.4 \%)$

$3(37.5 \%) \quad 5(83.3 \%)$

$6(33.3 \%) \quad 7(36.8 \%) \quad 5(62.5 \%) \quad 1(16.7 \%)$

3

$>3$

First-line therapy

Single agent

$4(6.2 \%)$

$1(5.6 \%)$

$2(10.5 \%)$

$3(16.7 \%)$

$1(5.2 \%)$

$-$

$3(4.6 \%)$

(notherapy

Triplet chemotherapy

Anti-angiogenic agent

Anti-EGFR

Therapies received

5-fluorouracile

Oxaliplatin

Irinotecan

Bevacizumab

Aflibercept

Cetuximab

Panitumumab

Intra-arterial therapy

Regorafenib

Number of lines

1

2

3

4

5

$3(4.6 \%)-$

$3(4.6 \%)$

$1(7.1 \%)$

$36(55.4 \%) \quad 7(50 \%)$

$10(15.4 \%) \quad 3(21.4 \%)$

$1(5.2 \%)$

$1(12.5 \%) \quad 1(16.7 \%)$

$2(11.1 \%)$

$12(66.7 \%)$

$3(16.7 \%)$

$7(87.5 \%)$

$5(83.3 \%)$

$10(52.6 \%)$

$5(62.5 \%) \quad 2(33.3 \%)$

$65(100 \%)$

$14(100 \%)$

$18(100 \%)$

$56(86.2 \%)$

$12(85.7 \%)$

$18(100 \%)$

$19(100 \%)$

$8(100 \%) \quad 6(100 \%)$

$53(81.5 \%)$

$9(64.3 \%)$

$15(83.3 \%)$

$15(83.3 \%)$

$16(84.2 \%)$

$7(87.5 \%$

$3(50 \%)$

$49(75.4 \%) \quad 9(64.3 \%)$

$17(89.5 \%)$

$8(100 \%)$

$4(66.7 \%)$

$15(23.1 \%) \quad 3(21.4 \%)$

$5(26.3 \%)$

$5(62.5 \%)$

$4(66.7 \%)$

$18(27.7 \%) \quad 5(35.7 \%)$

$6(33.3 \%)$

$2(25 \%)$

$2(33.3 \%)$

$11(16.9 \%) \quad 1(7.1 \%)$

$9(13.8 \%) \quad 1(7.1 \%)$

$(16.7 \%)$

$6(33.3 \%)$

$6(33.3 \%)$

$4(21.1 \%)$

$3(37.5 \%)$

$1(16.7 \%)$

$3(15.8 \%)$

$(16.7 \%)$

$10(15.4 \%)$

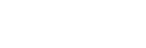

$2(11.1 \%)$

$5(27.8 \%)$

$25(38.5 \%) \quad 8(57.1 \%)$

$4(22.2 \%)$

$\begin{array}{lll}8(12.3 \%) & 1(7.1 \%) & 3(16.7 \%) \\ 6(9.2 \%) & - & 3(16.7 \%)\end{array}$

$2(10.5 \%)$

$9(47.4 \%)$

$3(15.8 \%)$

$3(15.8 \%)$

$2(10.5 \%)$

$-$

$1(12.5 \%) \quad-$

$\begin{array}{ll}- & 2(33.3 \%) \\ 2(25 \%) & 1(16.7 \%) \\ 5(62.5 \%) & 2(33.3 \%) \\ - & 1(16.7 \%)\end{array}$

$1(12.5 \%) \quad-$ 
Values are expressed as $n(\%)$ or means \pm standard deviation.

ETS early tumor shrinkage, $P R$ partial response, $S D$ stable disease tumor-size evolution 8 weeks after starting first-line chemotherapy (fl) between 0 and $+19 \%\left(\mathrm{SD}_{\mathrm{fl}}+\right)$ or 0 and $-29 \%\left(\mathrm{SD}_{\mathrm{fl}}-\right)$, PD progressive disease, MSS microsatellite stability, MSI microsatellite instability, EGFR epidermal growth-factor receptor. 
Table 2 Overall survival (OS), progression-free survival (PFS) and tumor burdens at endpoint and death

\begin{tabular}{lllllll}
\hline Characteristic & Total & $\mathrm{ETS}_{\mathrm{fl}}$ & $\mathrm{PR}_{\mathrm{fl}}$ & $\mathrm{SD}_{\mathrm{fl}}-$ & $\mathrm{SD}_{\mathrm{fl}}+$ & $\mathrm{PD}_{\mathrm{fl}}$ \\
\hline $\begin{array}{l}\text { Number of patients } \\
\text { Death }\end{array}$ & 65 & 14 & 18 & 19 & 8 & 6 \\
$\begin{array}{l}\text { Target lesions }>100 \% \text { at } \\
\text { Endpoint }\end{array}$ & $49(75.4 \%)$ & $6(42.9 \%)$ & $13(72.2 \%)$ & $18(94.7 \%)$ & $8(100 \%)$ & $4(66.7 \%)$ \\
$\quad$ & $30(46.2 \%)$ & $2(14.3 \%)$ & $6(33.3 \%)$ & $10(52.6 \%)$ & $7(87.5 \%)$ & $5(83.3 \%)$ \\
Death & $26(53.1 \%)$ & $1(16.7 \%)$ & $5(38.5 \%)$ & $9(50 \%)$ & $7(87.5 \%)$ & $4(100 \%)$ \\
OS, months & $22.7(20.6-27.0)$ & $33.3(12.6-47.2)$ & $24.3(18.6-32.6)$ & $20.8(17.2-24.7)$ & $22.7(15.7-37.7)$ & $14.1(1.5-35.7)$ \\
PFS, months & $8.6(9.2-14.3)$ & $15(9.8-24.2)$ & $9.1(7.6-16.5)$ & $7.2(5.9-14.0)$ & $4.7(1.2-19.5)$ & $2.9(4.9-15.3)$ \\
\hline
\end{tabular}

Results are expressed as $n(\%)$ or survival median (95\% confidence interval). Between-group differences were significant for OS ( $p=0.03)$, but not for $\operatorname{PFS}(p=0.3)$.

$E T S_{f l}$ early tumor shrinkage, $f l$ first-line chemotherapy, $P R_{f l}$ partial response, $S D$ stable disease tumor-size evolution between 0 and $+19 \%\left(S D_{f l}+\right)$ or 0 and $-29 \%\left(S D_{-f l}\right), P D_{f l}$ progressive disease, expressed as the percentage variation from the baseline $100 \%$ with return to that visual level. 
Table 3 Response and disease control rates after second- and third-line treatments, according to initial tumor-response group

\begin{tabular}{llllll}
\hline Parameter & $\mathrm{ETS}_{\mathrm{fl}}$ & $\mathrm{PR}_{\mathrm{fl}}$ & $\mathrm{SD}_{\mathrm{fl}}-$ & $\mathrm{SD}_{\mathrm{fl}}+$ & $\mathrm{PD}_{\mathrm{fl}}$ \\
\hline Second line, $n$ & 8 & 13 & 18 & 7 & 3 \\
Response rate & $3(37.5 \%)$ & $1(7.7 \%)$ & $1(5.6 \%)$ & $0(0 \%)$ & $0(0 \%)$ \\
Disease control rate & $8(100 \%)$ & $12(92.3 \%)$ & $14(77.8 \%)$ & $6(85.7 \%)$ & $1(33.3 \%)$ \\
Third line, $n$ & 3 & 11 & 12 & 4 & 2 \\
Response rate & $0(0 \%)$ & $2(18.2 \%)$ & $2(16.7 \%)$ & $0(0 \%)$ & $0(0 \%)$ \\
Disease control rate & $3(100 \%)$ & $8(72.7 \%)$ & $11(91.7 \%)$ & $3(75 \%)$ & $2(100 \%)$ \\
\hline
\end{tabular}

ETS early tumor shrinkage, $f l$ to first-line chemotherapy, $P R$ partial response, $S D$ stable disease tumor-size evolution between 0 and $+19 \%\left(S D_{f l}+\right)$ or 0 and $-29 \%\left(S D_{f \vdash}\right)$, or $P D$ progressive disease, expressed as the percentage variation from the baseline $100 \%$ with return to that visual level. 
ESM Table E1. PFS and OS, according to ETS status and reported ETS thresholds

\begin{tabular}{|c|c|c|c|c|c|c|c|c|c|}
\hline Study & Chemotherapy regimen & $\begin{array}{l}\text { ETS } \\
\text { definition }\end{array}$ & $\begin{array}{l}\text { Patients } \\
\text { with ETS } \\
(\%)\end{array}$ & $\begin{array}{l}\text { PFS with } \\
\text { ETS } \\
\text { (months) }\end{array}$ & $\begin{array}{l}\text { PFS without } \\
\text { ETS } \\
\text { (months) }\end{array}$ & $\triangle \mathrm{PFS}$ & $\begin{array}{l}\text { OS with } \\
\text { ETS } \\
\text { (months) }\end{array}$ & $\begin{array}{l}\text { OS without } \\
\text { ETS } \\
\text { (months) }\end{array}$ & $\Delta \mathrm{OS}$ \\
\hline \multirow[t]{2}{*}{$\mathrm{BOND}, 2009^{\mathrm{a}}$} & Irinotecan + cetuximab & $10 \%$ & NR & 7.1 & 1.6 & 5.5 & 12 & 7.4 & 4.6 \\
\hline & Cetuximab & & NR & 5.1 & 1.4 & 3.7 & NR & NR & NR \\
\hline \multirow[t]{2}{*}{ ACCORD 13, 2011} & FOLFIRI + bevacizumab & $\geq 20 \%$ & NR & 10 & 9 & 1 & 33 & 22 & 11 \\
\hline & XELIRI + bevacizumab & & NR & NR & NR & NR & NR & NR & NR \\
\hline FIRE-1, 2013 ${ }^{\mathrm{b}}$ & FUFIRI + mIROX & $\geq 20 \%$ & $47 \%$ & 9.9 & 6.1 & 3.8 & 27.5 & 17.8 & 9.7 \\
\hline \multirow{2}{*}{ CRYSTAL, $2013^{\mathrm{c}}$} & FOLFIRI + cetuximab & $\geq 20 \%$ & $62 \%$ & 14.1 & 7.3 & 6.8 & 30 & 18.6 & 11.4 \\
\hline & FOLFIRI & & $51 \%$ & 9.7 & 7.4 & 2.3 & 24.1 & 18.6 & 5.5 \\
\hline \multirow[t]{2}{*}{ OPUS, $2013^{\mathrm{c}}$} & FOLFOX + cetuximab & $\geq 20 \%$ & $69 \%$ & 11.9 & 5.7 & 6.2 & 26 & 15.7 & 10.3 \\
\hline & FOLFOX & & $46 \%$ & 7.2 & 7.2 & 0 & 21.6 & 17.8 & 3.8 \\
\hline CIOX, 2013 & $\begin{array}{l}\text { CAPIRI/CAPOX + } \\
\text { cetuximab }\end{array}$ & $\geq 20 \%$ & $59 \%$ & 8.9 & 4.7 & 4.2 & 31.6 & 15.8 & 15.8 \\
\hline \multirow[t]{2}{*}{ Ye, 2015} & $\begin{array}{l}\text { FOLFIRI/mFOLFOX6 + } \\
\text { cetuximab }\end{array}$ & $\geq 20 \%$ & NR & 11.8 & 4.8 & 7 & 38 & 18.7 & 19.3 \\
\hline & FOLFIRI/mFOLFOX6 & & NR & 8 & 4.6 & 3.4 & 30.6 & 17.7 & 12.9 \\
\hline \multirow[t]{2}{*}{ PRIME, $2015^{\mathrm{c}}$} & FOLFOX4 + panitumumab & $\geq 20 \%$ & $72 \%$ & 13.6 & 6.7 & 6.9 & 32.5 & 12.6 & 19.9 \\
\hline & FOLFOX & & $59 \%$ & 14.9 & 9.3 & 5.6 & 34.5 & 18.2 & 16.3 \\
\hline \multirow[t]{2}{*}{ TRIBE, 2015} & $\begin{array}{l}\text { FOLFOXIRI + } \\
\text { bevacizumab }\end{array}$ & $\geq 20 \%$ & $64 \%$ & 8.8 & 7.2 & 1.6 & 31.9 & 21.9 & 10 \\
\hline & FOLFIRI + bevacizumab & & NR & NR & NR & NR & NR & NR & NR \\
\hline JACCRO-CC05, 2016 & FOLFOX + cetuximab & $\geq 20 \%$ & $80 \%$ & 11.3 & 3.7 & 7.6 & 42.8 & 9 & 33.8 \\
\hline \multirow[t]{2}{*}{ FIRE-3, 2016} & FOLFIRI + cetuximab & $\geq 20 \%$ & $68 \%$ & 9.7 & 5.8 & 3.9 & 38.3 & 20.5 & 17.8 \\
\hline & FOLFIRI + bevacizumab & & $49 \%$ & 11.7 & 8.3 & 3.4 & 31.9 & 21.2 & 10.7 \\
\hline \multirow{3}{*}{ PEAK, 2017} & FOLFOX6 + bevacizumab & $\geq 20 \%$ & $62 \%$ & 11.3 & 9.5 & 1.8 & 32.5 & 21.8 & 10.7 \\
\hline & & $\geq 30 \%$ & $45 \%$ & 11.1 & 9.7 & 1.4 & 35.1 & 23.9 & 11.2 \\
\hline & FOLFOX6 + panitumumab & & $75 \%$ & 13.1 & 9.8 & 3.3 & 43.4 & 21.2 & 22.2 \\
\hline
\end{tabular}

ETS early tumor shrinkage, $P F S$ progression-free survival, $\triangle$ change, $O S$ overall survival, CAPIRI capecitabine + irinotecan, CAPOX capecitabine + oxaliplatin, FOLFIRI 5-fluorouracil + irinotecan + leucovorin, FOLFOX folinic acid + 5-fluorouracil + oxaliplatin, FOLFOX-4 5-fluorouracil + leucovorin + oxaliplatin, FUFIRI 5-fluorouracil + folinic acid + irinotecan; mFOLFOX6 modified FOLFOX, mIROX modified irinotecan plus oxaliplatin, $X E L I R I$ capecitabine + irinotecan, $N R$ not reported.

${ }^{\mathrm{a}}$ Time-to-progression used. 
${ }^{\mathrm{b}}$ ETS was assessed at 7 weeks.

${ }^{\mathrm{c}}$ Evaluation of patients with $K R A S$ wild-type tumors.

${ }^{\mathrm{d}}$ ETS was assessed at 6 weeks. 